\title{
A System of Quasi Variational Inequalities and its Application to Reaction Diffusion Equations
}

By

\author{
Koichiro NAITO*
}

\section{§1. Introduction}

To control a flow and exchange of material through its boundary is the most important problem for the organism to maintain the order on the dynamic equilibrium. Mathematically this complicated control through the boundary or membrane of the organism is considered as various types of boundary conditions. For instance, by using the methods of variational inequalities (abr. V.I.) or quasi variational inequalities (abr. Q.V.I.), Duvaut and Lions studied the unilateral problem in [3] such that

$$
-\Delta u+k u=0 \quad \text { in } \Omega
$$

$$
u \frac{\partial u}{\partial n}=0, \frac{\partial u}{\partial n} \geqq 0, u \geqq 0 \quad \text { on } \quad \partial \Omega
$$

where $u$ describes the concentration of a chemical substance in the domain $\Omega$ and $\frac{\partial}{\partial n}$ is the outward normal derivative. The boundary conditions (1.2) mean that the flow through the boundary into the domain is shut up on the place where $\left.u\right|_{\partial \Omega}>0$ and, on the other hand, the substance is allowed to enter into the domain on the place where $\left.u\right|_{\partial \Omega}=0$. In the last part of this paper we consider the boundary conditions called "a priori feedback control" model which was introduced by Lions [8];

Communicated by S. Matsuura, April 20, 1981. Revised November 17, 1984.

* Department of Information Sciences, Tokyo Institute of Technology, Oh-okayama, Meguro-ku, Tokyo 152, Japan. 
(1.3) $\begin{cases}\frac{\partial u}{\partial n}=0 & \text { on } \partial \Omega_{+}=\left\{y \in \partial \Omega:\left.u\right|_{\partial \Omega}(y)>Q\left(\left.u\right|_{\partial \Omega}\right)(y)\right\} \\ \frac{\partial u}{\partial n}=M & \text { on } \partial \Omega_{-}=\left\{y \in \partial \Omega:\left.u\right|_{\partial \Omega}<Q\left(\left.u\right|_{\partial \Omega}\right)\right\} \\ 0 \leqq \frac{\partial u}{\partial n} \leqq M & \end{cases}$

where $Q: L^{2}(\partial \Omega) \rightarrow L^{2}(\partial \Omega)$ is continuous and uniformly bounded. In the case of Lions [8] $Q\left(\left.u\right|_{\partial \Omega}\right)(y)$ is a constant function defined by

$$
Q\left(\left.u\right|_{\partial \Omega}\right)(y) \equiv \operatorname{mean}\left[\left.u\right|_{\partial \Omega}\right]=\left.\frac{1}{m(\partial \Omega)} \int_{\partial \Omega} u\right|_{\partial \Omega}(y) d y
$$

which means the substrate is allowed to enter into the domain through the part of the boundary where the concentration is lower than the average and on the part where the concentration is higher than the average the flow is shut up. When $Q\left(\left.u\right|_{\partial \Omega}\right) \equiv \varepsilon$ and $\varepsilon>0$ is small enough, the unilateral problem is approximated by (1.3).

In this paper first we study a system of Q.V.I. General forms of Q. V.I. were introduced and studied by Mosco in [10] and Mossino also applied a general theory of $Q$. V.I. to the equations from Plasma Physics in [11]. She used the function type of Q. V.I. such that

$$
F(u, u) \leqq F(v, u) \quad \text { for every } v \in U,
$$

that is,

$$
0 \in \partial F(u, u), \quad u \in U
$$

where $U, V$ are reflexive Banach spaces and $F: U \times V \rightarrow R$ and the subdifferentials are taken with respect to the first variables.

To analyze reaction-diffusion systems we introduce a system of Q. V.I.

$$
\left\{\begin{array}{l}
0 \in \partial F_{1}(s, p) \\
0 \in \partial F_{2}(p, s)
\end{array}\right.
$$

and we prove the existence of a pair of solutions by applying the method of Mossino. Next we consider a system of reaction-diffusion equations which was mathematically formulated by Kernevez [7] or Banks [1] as an active transport model of double-layered membranes. We construct convex functionals. By applying the theory of Rockafellar about integrals of convex functions we calculate the subdifferentials of these functionals and show the existence of solutions in equilibrium 
as minimal points of these convex functionals.

We study this reaction-diffusion system with the following two types of boundary conditions:

(1) The Dirichlet boundary conditions - We assume that the boundary is absorptive or chemical components are dissolved on the boundary.

(2) The Neumann boundary conditions-There exists the flux through the boundary which is controled by the concentration of the other reacting chemical component. In this case we assume that the chemical components are dissolved at the rate proportional to their concentration in the domain.

It should be remarked that we can show the existence of the stationary solutions when the components are dissolved in the domain or on the boundary. These conditions of dissolution yield the saturated states chemically and also yield the coercive conditions mathematically. In our cases the coercivity is the most essential condition for the existence of the stationary solutions. When the coercive conditions are satisfied, we obtain the solutions as minimal points of convex functionals.

In $\S 2$ we study a system of Q.V.I. and we show the existence of solutions. By applying this Q.V.I. system we show the existence of stationary solutions of reaction-diffusion equations with Dirichlet boundary conditions in $\S 3$ and with Neumann boundary conditions in $\S 4$ and, especially, we treat "a priori feedback control" problem in $\S 5$.

\section{§2. System of General Q. $\mathrm{V} . \mathbb{I}$.}

Let $U, V$ be real reflexive Banach spaces and assume that $U$ is compactly imbedded in $V$, that is, (1) $U$ is a vector subspace of $V$, and (2) the identity operator $I$ defined on $U$ into $V$ is continuous and compact.

Let $M_{1}, M_{2}$ be nonempty closed convex subsets of $U$ and $F_{1}, F_{2}$ be functions defined on $U \times V$ with values in $(-\infty,+\infty]$, satisfying the following conditions:

(i. a) $F_{1}(\cdot, \cdot)$ is lower semi-continuous (abr. 1.s. c.) on $M_{1}$ (relative weak topology of $U) \times M_{2}$ (relative strong topology of $V$ );

(i. b) $F_{2}(\cdot, \cdot)$ is 1 . s. c. on $M_{2}$ (relative weak top. of $U$ ) $\times M_{1}$ (relative strong top. of $V$ ); 
(i. c) for each $s \in M_{1}, F_{1}\left(s,{ }^{\circ}\right)$ is continuous on $M_{2}$ (relative strong top. of $V$ ) ;

(i. d) for each $q \in M_{2}, F_{2}\left(q,{ }^{\circ}\right)$ is continuous on $M_{1}$ (relative strong top. of $V$ );

(ii. a) for each $p \in M_{2}, F_{1}(\cdot, p)$ is proper, strictly convex;

(ii. b) for each $r \in M_{1}, F_{2}(\cdot, r)$ is proper, strictly convex;

(iii. a) $F_{1}$ is coercive on $M_{1}$ in the following sense, there exists an element $s_{0} \in M_{1}$ such that, if $s \in M_{1}$ and $\|s\|_{U} \rightarrow+\infty$, then $F_{1}(s, p)-F_{1}\left(s_{0}, p\right) \rightarrow+\infty$, uniformly w.r.t. $p$ in $M_{2}$;

(iii. b) $F_{2}$ is coercive on $M_{2}$, that is, there exists an element $q_{0}$ in $M_{2}$ such that, if $q \in M_{2}$ and $\|q\|_{U} \rightarrow+\infty$, then $F_{2}(q, r)-$ $F_{2}\left(q_{0}, r\right) \rightarrow+\infty$, uniformly w.r.t. $r$ in $M_{1}$.

Theorem 2.1. Under the above assumptions, we have a pair of solutions $(\xi, \eta)$ in $M_{1} \times M_{2}$ of Q.V.I. given by

$$
\begin{cases}F_{1}(\xi, \eta) \leqq F_{1}(s, \eta) & \text { for all } s \text { in } M_{1} \\ F_{2}(\eta, \xi) \leqq F_{2}(q, \xi) & \text { for all } q \text { in } M_{2} .\end{cases}
$$

We use the following lemmas for the proof of Theorem.

Lemma 2.1. For each $p \in M_{2}$, there exists a unique element $s_{p}$ in $M_{1}$;

$$
F_{1}\left(s_{p}, p\right) \leqq F_{1}(s, p) \quad \text { for all } s \text { in } M_{1} \text {, }
$$

and for each $r \in M_{1}$, there exists a unique element $q_{r}$ in $M_{2}$;

$$
F_{2}\left(q_{r}, r\right) \leqq F_{2}(q, r) \quad \text { for all } q \text { in } M_{2} \text { 。 }
$$

Proof. It can be easily proved by the results of Ekeland-Temam [4] or Barbu [2], because $F_{1}(\cdot, p)$ is proper, strictly convex, coercive, and 1. s. c. with the weak topology of $U$ for each $p$ in $M_{2}$ and also $M_{1}$ is closed convex in $U$. Furthermore, $F_{2}, M_{2}$ have same properties.

Q. E. D.

Lemma. 2.2。 We define mappings $T_{1}: M_{2} \rightarrow M_{1}, \quad T_{2}: \quad M_{1} \rightarrow M_{2}$ by $T_{1} p=s_{p}$ for each $p$ in $M_{2}$ and $T_{2} r=q_{r}$ for each $r$ in $M_{1}$. Then $T_{1}$ and $T_{2}$ are continuous with the strong topology of $V$ and

$$
\sup _{p \in M \mathbf{2}}\left\|T_{1} p\right\|_{U}=K_{1}<+\infty
$$

and

$$
\sup _{r \in M_{1}} \|\left. T_{2} r\right|_{U}=K_{2}<+\infty
$$


Proof. $\quad T_{1}, T_{2}$ are well defined by Lemma 2.1. From the definition of $T_{1}$, we have

$$
F_{1}\left(T_{1} p, p\right)-F_{1}\left(s_{0}, p\right) \leqq 0
$$

where $s_{0}$ is the element defined in the coercive condition (iii)。 By the coerciveness we can easily see that

$$
\sup _{p \in M_{2}}\left\|T_{1} p\right\|_{U}=K_{1}<+\infty \text {. }
$$

Now we shall show the continuity of $T_{1}$. Consider a sequence $\left\{p_{n}\right\} \subset M_{2}$ convergent to $p_{1}$ in $M_{2}$ with the strong topology of $V$. Since $\left\|T_{1} p_{n}\right\|_{U} \leqq K_{1}$ and $M_{1}$ is closed convex in $U$, there exist $s_{1} \in M_{1}$ and a subsequence $\left\{p_{n_{j}}\right\}$ of $\left\{p_{n}\right\}$ such that

$$
T_{1} p_{n_{j}} \rightarrow s_{1} \text { in } M_{1} \text { with the weak top. of } U
$$

as $n_{j} \rightarrow+\infty$. Then we have

$$
T_{1} p_{n_{j}} \rightarrow s_{1} \text { in } M_{1} \text { with the strong top. of } V \text {, }
$$

because $U$ is compactly imbedded in $V$. From the definition of $T_{1}$ we have

$$
F_{1}\left(T_{1} p_{n_{j}}, p_{n_{j}}\right) \leqq F_{1}\left(s, p_{n_{j}}\right) \quad \text { for all } s \text { in } M_{1} \text {. }
$$

Since $F_{1}\left({ }^{\circ},{ }^{\circ}\right)$ is 1. s. c. and $F_{1}\left(s,{ }^{\circ}\right)$ is continuous on $M_{2}$ for each $s$ in $M_{1}$ in the sense of (i), we obtain

$$
\begin{aligned}
F_{1}\left(s_{1}, p_{1}\right) & \leqq \liminf _{n_{j} \rightarrow+\infty} F_{1}\left(T_{1} p_{n_{j}}, p_{n_{j}}\right) \\
& \leqq \liminf _{n_{j} \rightarrow+\infty} F_{1}\left(s, p_{n_{j}}\right) \\
& =F_{1}\left(s, p_{1}\right) \quad \text { for all } s \text { in } M_{1} .
\end{aligned}
$$

Since the minimum point is unique, $s_{1}=T_{1} p_{1}$. We can conclude that $T_{1} p_{n} \rightarrow T_{1} p_{1}$ in $M_{1}$ with the strong top. of $V$.

$\begin{array}{lll}\text { Similar arguments imply the results about } T_{2} & \text { Q. E. D. }\end{array}$

Proof of Theorem 2.1. Let $B=M_{2} \cap\left\{u \in U:\|u\|_{U} \leqq K_{2}\right\}$. Then $B$ is a nonempty weakly compact convex subset of $U$ by Lemma 2.2 and the reflexivity of $U$. Furthermore, $B$ is compact convex with the strong top. of $V$, because $U$ is compactly imbedded in $V$.

Since $T_{1} M_{2} \subset M_{1}, T_{2} M_{1} \subset B$, and the composition $T_{2} \circ T_{1}$ is continuous on $M_{2}$ with the strong top. of $V$ by applying Lemma 2.2, there exists a fixed point $\eta$ in $B \subset M_{2}$, i.e., $T_{2} \circ T_{1} \eta=\eta$ by Schauder's fixed 
point theorem. Let $\xi=T_{1} \eta$, then we have a pair of $Q$. V.I. solutions $(\xi, \eta)$ in $M_{1} \times M_{2}$ :

$$
F_{1}(\xi, \eta) \leqq F_{1}(s, \eta) \quad \text { for all } s \text { in } M_{1}
$$

and

$$
F_{2}(\eta, \xi) \leqq F_{2}(q, \xi) \quad \text { for all } q \text { in } M_{2} \text {. }
$$

Q. E. D.

Remark. To show the uniqueness of solutions of this system is an interesting problem, but the assumptions for the uniqueness will be complicated, because we need the arguments about the uniqueness in the Schauder's fixed point theorem [6].

\section{§ 3. Dirichlet Boundary Conditions}

Let the cell $\Omega$ be a bounded open subset in $R^{n}$ with the sufficiently smooth boundary $\Gamma=\partial \Omega$. Assume that the organs $\Omega_{1}, \Omega_{2}$ are measurable subsets of $\Omega$ and $\Omega_{1} \cap \Omega_{2}=\phi$. Enzymes $E_{1}, E_{2}$ are embedded in $\Omega_{1}$, $\Omega_{2}$, respectively. We have coupled reactions between two chemical components $S$ and $P$. In $\Omega_{1}$ we have the irreversible reaction with competitive inhibition;

$$
S \stackrel{E_{1}}{\longrightarrow} P
$$

where $S$ is the substrate of $E_{1}$ and the product $P$ is an inhibitor of this reaction. In $\Omega_{2} P$ is the substrate for the enzyme $E_{2}$ which catalyzes the irreversible reaction;

$$
P \stackrel{E_{2}}{\longrightarrow} S \text {. }
$$

Here we consider a system of reaction-diffusion equations with the absorptive boundary $\Gamma$ which can dissolve or absorb the substrate and the product. We assume that the diffusion coefficients of $S$ and $P$ are in the ratio of $1: \lambda$. Then, in the stationary case, this model is described by the following system of the differential equations. (See [1] or [7] for further details.)

$$
\left\{\begin{array}{l}
-\Delta s+\chi_{1} \frac{\sigma_{1} s^{+}}{1+\alpha p^{+}+s^{+}}-\chi_{2} \frac{\sigma_{2} p^{+}}{1+\beta p^{+}}-f_{1}=0 \\
-\lambda \Delta p+\chi_{2} \frac{\sigma_{2} p^{+}}{1+\beta p^{+}}-\chi_{1} \frac{\sigma_{1} s^{+}}{1+\alpha p^{+}+s^{+}}-f_{2}=0
\end{array}\right.
$$




$$
\left\{\begin{array}{l}
\left.s\right|_{\Gamma}=0 \\
\left.p\right|_{\Gamma}=0
\end{array}\right.
$$

where

$$
\mathcal{J}=\sum_{i=1}^{n} \frac{\partial^{2}}{\partial x_{i}^{2}}
$$

in the sense of distributions,

$$
\begin{aligned}
& \chi_{1}(x)= \begin{cases}1 & \text { if } x \in \Omega_{1} \\
0 & \text { if } x \notin \Omega_{1},\end{cases} \\
& \chi_{2}(x)= \begin{cases}1 & \text { if } x \in \Omega_{2} \\
0 & \text { if } x \notin \Omega_{2},\end{cases}
\end{aligned}
$$

$s(x)$ is the concentration of $S$ at $x \in \Omega$,

$p(x)$ is the concentration of $P$ at $x \in \Omega$,

$p^{+}=\max (p, 0), s^{+}=\max (s, 0)$,

$\sigma_{1}, \sigma_{2}, \alpha, \beta$, and $\lambda$ are positive constants,

$f_{1}(x)$ is the supply of $S$ at $x \in \Omega$,

$f_{2}(x)$ is the supply of $P$ at $x \in \Omega, \quad f_{1}, f_{2} \in L^{2}(\Omega)$,

and we take a suitable unity of concentration.

Now we deal with the problem (3.1)-(3.2) by using the results of $\S 2$. Consider the functions $\phi_{1}, \phi_{2}, \phi_{1}$, and $\phi_{2}: R \times R \rightarrow R$ defined by

$$
\begin{aligned}
& \phi_{1}(a, b)=\sigma_{1}\left\{a^{+}-\left(1+\alpha b^{+}\right) \log \left(1+a^{+}+\alpha b^{+}\right)\right\}, \\
& \phi_{2}(a, b)=-\sigma_{2} \frac{b^{+}}{1+\beta b^{+}} a, \\
& \phi_{1}(a, b)=-\sigma_{1}\left\{\frac{b^{+}}{1+b^{+}}\left(a-a^{+}\right)+\frac{b^{+}}{\alpha} \log \left(1+\alpha a^{+}+b^{+}\right)\right\}, \\
& \phi_{2}(a, b)=\phi_{2}(a)=\frac{\sigma_{2}}{\beta}\left\{a^{+}-\frac{1}{\beta} \log \left(1+\beta a^{+}\right)\right\} .
\end{aligned}
$$

Let $G_{1}, G_{2}$ be real valued functions defined on $H_{0}^{1}(\Omega) \times L^{2}(\Omega)$ :

$$
\begin{aligned}
G_{1}(s, p)= & \frac{1}{2} \int_{\Omega}|\nabla s|^{2} d x+\int_{\Omega} \chi_{1}(x) \phi_{1}(s(x), p(x)) d x \\
& +\int_{\Omega} \chi_{2}(x) \phi_{2}(s(x), p(x)) d x-\int_{\Omega} f_{1} s d x, \\
G_{2}(q, r)= & \frac{1}{2} \lambda \int_{\Omega}|\nabla q|^{2} d x+\int_{\Omega} \chi_{2}(x) \phi_{2}(q(x), r(x)) d x
\end{aligned}
$$




$$
+\int_{\Omega} \chi_{1}(x) \phi_{1}(q(x), r(x)) d x-\int_{\Omega} f_{2} q d x
$$

The integrals are well defined, because the composition of a Borel measurable function and a Lebesgue measurable function is Lebesgue measurable and also $\phi_{i}(s(x), p(x)), \phi_{i}(q(x), r(x)), i=1,2$, are summable by using the inequalities

$$
\frac{t}{1+t} \leqq \log (1+t) \leqq t \quad \text { for all } t \geqq 0
$$

Theorem 3. 1. There exists a pair of solutions $(s, p)$ in $H_{0}^{1}(\Omega) \times H_{0}^{1}(\Omega)$, which satisfies the system of $Q . I^{\top} . I$. given by

$$
\begin{cases}G_{1}(s, p) \leqq G_{1}(u, p) & \text { for all } u \in H_{0}^{1}(\Omega) \\ G_{2}(p, s) \leqq G_{2}(v, s) & \text { for all } v \in H_{0}^{1}(\Omega) .\end{cases}
$$

Proof. In view of Theorem 2. 1, let $U=M_{1}=M_{2}=H_{0}^{1}(\Omega), V=L^{2}(\Omega)$, $F_{1}=G_{1}$, and $F_{2}=G_{2}$, then it is sufficient to show that $G_{1}$ and $G_{2}$ satisfy the conditions (i), (ii) and (iii) in $\S 2$.

(i): Consider a sequence $\left\{s_{n}\right\} \subset H_{0}^{1}(\Omega)$ convergent to an element $s_{1}$ in $H_{0}^{1}(\Omega)$ with the weak topology of $H_{0}^{1}(\Omega)$ and a sequence $\left\{p_{n}\right\} \subset L^{2}(\Omega)$ convergent to $p_{1}$ with the strong topology of $L^{2}(\Omega)$. Since $H_{0}^{1}(\Omega)$ is compactly imbedded in $L^{2}(\Omega), s_{n} \rightarrow s_{1}$ in $L^{2}(\Omega)$ strongly. For an element $v$ in $L^{2}(\Omega)$, we define $v^{+}, v^{-}$by $v^{+}=\max (v, 0), v^{-}=-\min (v, 0)$. It can be easily shown that

$$
s_{n}^{+} \rightarrow s_{1}^{+}, \quad s_{n}^{-} \rightarrow s_{1}^{-} \quad \text { in } L^{2}(\Omega), \text { strongly, }
$$

and

$$
p_{n}^{+} \rightarrow p_{1}^{+}, \quad p_{n}^{-} \rightarrow p_{1}^{-} \quad \text { in } L^{2}(\Omega), \text { strongly. }
$$

We claim that, as $n \rightarrow+\infty$,

$$
\int_{\Omega} \chi_{1}(x) \phi_{1}\left(s_{n}, p_{n}\right) d x \rightarrow \int_{\Omega} \chi_{1}(x) \phi_{1}\left(s_{1}, p_{1}\right) d x
$$

and

$$
\int_{\Omega} \chi_{2}(x) \phi_{2}\left(s_{n}, p_{n}\right) d x \rightarrow \int_{\Omega} \chi_{2}(x) \phi_{2}\left(s_{1}, p_{1}\right) d x,
$$

which is the direct consequence of the following calculations.

$$
\begin{aligned}
\left|\int_{\Omega_{1}} \phi_{1}\left(s_{n}, p_{n}\right)-\int_{\Omega_{1}} \phi_{1}\left(s_{1}, p_{1}\right)\right|= & \sigma_{1} \mid \int_{\Omega_{1}}\left\{s_{n}^{+}-\left(1+\alpha p_{n}^{+}\right) \log \left(1+s_{n}^{+}+\alpha p_{n}^{+}\right)\right\} \\
& -\int_{\Omega_{1}}\left\{s_{1}^{+}-\left(1+\alpha p_{1}^{+}\right) \log \left(1+s_{1}^{+}+\alpha p_{1}^{+}\right)\right\} \mid
\end{aligned}
$$




$$
\begin{aligned}
\leqq & \sigma_{1} \int\left|s_{n}^{+}-s_{1}^{+}\right|+\sigma_{1} \int \mid\left(1+\alpha p_{n}^{+}\right) \log \left(1+s_{n}^{+}+\alpha p_{n}^{+}\right) \\
& \quad-\left(1+\alpha p_{1}^{+}\right) \log \left(1+s_{1}^{+}+\alpha p_{1}^{+}\right) \mid \\
\leqq & \sigma_{1} \int\left|s_{n}^{+}-s_{1}^{+}\right|+\sigma_{1} \int\left|\alpha\left(p_{n}^{+}-p_{1}^{+}\right) \log \left(1+s_{n}^{+}+\alpha p_{n}^{+}\right)\right| \\
& \quad+\sigma_{1} \int\left(1+\alpha p_{1}^{+}\right)\left|\log \left(1+s_{n}^{+}+\alpha p_{n}^{+}\right)-\log \left(1+s_{1}^{+}+\alpha p_{1}^{+}\right)\right| \\
\leqq & \sigma_{1} \int\left|s_{n}^{+}-\mathrm{s}_{1}^{+}\right|+\sigma_{1} \alpha \int\left|p_{n}^{+}-p_{1}^{+}\right|\left|s_{n}^{+}+\alpha p_{n}^{+}\right| \\
& +\sigma_{1} \int\left(1+\alpha p_{1}^{+}\right) \frac{\left|s_{n}^{+}-s_{1}^{+}+\alpha p_{n}^{+}-\alpha p_{1}^{+}\right|}{1+s_{1}^{+}+\alpha p_{1}^{+}} \\
\leqq & c\left\|s_{n}^{+}-s_{1}^{+}\right\|_{L^{2}}+c\left\|p_{n}^{+}-p_{1}^{+}\right\|_{L^{2}}\left\{\left\|s_{n}^{+}\right\|_{L^{2}}+\alpha\left\|p_{n}^{+}\right\|_{L^{2}}\right\} \\
& +c\left\{\left\|s_{n}^{+}-s_{1}^{+}\right\|_{L^{2}}+\alpha\left\|p_{n}^{+}-p_{1}^{+}\right\| L_{L^{2}}\right\}
\end{aligned}
$$

where we use the inequality (3.3),

$$
\begin{aligned}
\mid \int_{\Omega_{2}} \phi_{2}\left(s_{n}, p_{n}\right) & -\int_{\Omega_{2}} \phi_{2}\left(s_{1}, p_{1}\right)\left|=\sigma_{2}\right| \int_{\Omega_{2}}\left\{\frac{p_{n}^{+}}{1+\beta p_{n}^{+}} s_{n}-\frac{p_{1}^{+}}{1+\beta p_{1}^{+}} s_{1}\right\} \mid \\
& \leqq \sigma_{2} \int\left|\frac{p_{n}^{+}}{1+\beta p_{n}^{+}}\left(s_{n}-s_{1}\right)\right|+\sigma_{2} \int\left|\frac{p_{n}^{+}}{1+\beta p_{n}^{+}}-\frac{p_{1}^{+}}{1+\beta p_{1}^{+}}\right|\left|s_{1}\right| \\
& \leqq \sigma_{2} \int \frac{1}{\beta}\left|s_{n}-s_{1}\right|+\sigma_{2} \int \frac{\left|p_{n}^{+}-p_{1}^{+}\right|\left|s_{1}\right|}{\left(1+\beta p_{n}^{+}\right)\left(1+\beta p_{1}^{+}\right)} \\
& \leqq c|| s_{n}-s_{1} \|_{L^{2}}+\left.c\right|_{1} ^{\prime} p_{n}^{+}-\left.p_{1}^{+1}\right|_{L^{2}}
\end{aligned}
$$

where $c$ is a positive suitable constant which is independent of $n$ and we use the abbreviation

$$
\int=\int_{\Omega} d x
$$

Furthermore, we have

$$
\int f_{1} s_{n} \rightarrow \int f_{1} s_{1} \quad \text { as } n \rightarrow+\infty
$$

and also $\int|\nabla \cdot|^{2}$ is weakly 1 . s. c. on $H_{0}^{1}(\Omega)$. Similarly, we can show the lower semi-continuity of $G_{2}\left(\bullet^{\circ},{ }^{\circ}\right)$ and the continuity of $G_{2}\left(q,{ }^{\circ}\right)$ for each $q$ in $M_{2}$.

(ii) : The strictly convex property is easily shown by using the fact that $\phi_{1}(\bullet, b), \phi_{2}(\bullet, b), \phi_{1}(\cdot, b)$ and $\psi_{2}(\cdot, b)$ are convex for each $b$ in $R$ and $\int|\cdot|^{2}$ is strictly convex.

(iii) : Let $s_{0}=0, q_{0}=0$. The following calculations show the coerciveness of $G_{1}, G_{2}$. 


$$
\begin{aligned}
G_{1}(s, p)-G_{1}(0, p)= & \frac{1}{2} \int|\nabla s|^{2}+\int \sigma_{1} \chi_{1}\left\{s^{+}-\left(1+\alpha p^{+}\right) \log \left(1+\alpha p^{+}+s^{+}\right)\right\} \\
& -\int \sigma_{2} \chi_{2} \frac{p^{+}}{1+\beta p^{+}} s-\int \sigma_{1} \chi_{1}\left\{-\left(1+\alpha p^{+}\right) \log \left(1+\alpha p^{+}\right)\right\}-\int f_{1} s \\
= & \frac{1}{2} \int|\nabla s|^{2}+\int \sigma_{1} \chi_{1}\left\{s^{+}-\left(1+\alpha p^{+}\right) \log \frac{1+s^{+}+\alpha p^{+}}{1+\alpha p^{+}}\right\} \\
& -\int \sigma_{2} \chi_{2} \frac{p^{+}}{1+\beta p^{+}} s-\int f_{1} s \\
\geqq & \frac{1}{2} \int|\nabla s|^{2}+\int \sigma_{1} \chi_{1}\left\{s^{+}-\left(1+\alpha p^{+}\right) \frac{s^{+}}{1+\alpha p^{+}}\right\} \\
& -\int \sigma_{2} \chi_{2} \cdot \frac{p^{+}}{1+\beta p^{+}} \cdot|s|-\int\left|f_{1}\right||s| \\
\geqq & \left.C_{1}|| s\right|_{H_{0}^{1}} ^{2}-\left.C_{2}|| s\right|_{H_{0}^{1}}, \quad G_{1}>0, C_{2}>0
\end{aligned}
$$

where we use Poincarés inequality and the fact that the injection $I: H_{0}^{1}(\Omega) \rightarrow L^{2}(\Omega)$ is continuous. Similarly, we have

$$
\begin{aligned}
G_{2}(q, r)-G_{2}(0, r)= & \frac{\lambda}{2} \int|\nabla q|^{2}+\int \frac{\sigma_{2}}{\beta} \chi_{2}\left\{q^{+}-\frac{1}{\beta} \log \left(1+\beta q^{+}\right)\right\} \\
& -\int \sigma_{1} \chi_{1}\left\{\frac{r^{+}}{1+r^{+}}\left(q-q^{+}\right)+\frac{r^{+}}{\alpha} \log \left(1+\alpha q^{+}+r^{+}\right)\right\} \\
& -\int f_{2} q+\int \sigma_{1} \chi_{1} \frac{r^{+}}{\alpha} \log \left(1+r^{+}\right) \\
\geqq & \frac{\lambda}{2} \int|\nabla q|^{2}+\int \frac{\sigma_{2}}{\beta} \chi_{2}\left\{q^{+}-\frac{1}{\beta} \cdot \beta q^{+}\right\} \\
& -\int \sigma_{1} \chi_{1}\left\{\frac{r^{+}}{1+r^{+}}\left(q-q^{+}\right)+\frac{r^{+}}{\alpha} \log \left(1+\frac{\alpha q^{+}}{1+r^{+}}\right)\right\}-\int f_{2} q \\
\geqq & \frac{\lambda}{2} \int|\nabla q|^{2}-\int \sigma_{1} \chi_{1}\left\{\frac{r^{+}}{1+r^{+}}\left(q-q^{+}\right)+\frac{r^{+} \cdot \alpha q^{+}}{\alpha\left(1+r^{+}\right)}\right\} \\
& -\int\left|f_{2}\right||q| \quad C_{3}|| q\left\|_{H_{0}^{1}}^{2}-C_{4}\right\| q \|_{H_{0}^{1}}, \quad C_{3}>0, C_{4}>0 .
\end{aligned}
$$

Q.E.D.

Definition 3.1. Let $X$ be a real Banach space with its dual $X^{*}$ and $(\cdot, \cdot)$ denote the dual pairing between $X^{*}$ and $X$. Given the proper convex function $f: X \rightarrow(-\infty,+\infty]$, the subdifferential of such a function is the generally multivalued mapping $\partial f: X \rightarrow X^{*}$ defined by 


$$
\partial f(x)=\left\{x^{*} \in X^{*}: f(u)-f(x) \geqq\left(x^{*}, u-x\right) \text { for }{ }^{\forall} u \in X\right\} .
$$

The elements $x^{*} \in \partial f(x)$ are called subgradients of $f$ at $x$.

Definition 3.2. Consider any function $f: X \rightarrow(-\infty,+\infty]$. The function $f^{*}: X^{*} \rightarrow[-\infty,+\infty]$ defined by

$$
f^{*}\left(x^{*}\right)=\sup \left\{\left(x^{*}, x\right)-f(x): x \in X\right\}
$$

is called the conjugate function of $f$.

We obtain the following Theorem from the definition of subdifferentials and Theorem 3.1.

Theorem 3.2. We have a solution $(s, p)$ in $H_{0}^{1}(\Omega) \times H_{0}^{1}(\Omega)$ of the following system;

$$
\left\{\begin{array}{l}
0 \in \partial G_{1}(s, p) \\
0 \in \partial G_{2}(p, s)
\end{array}\right.
$$

where we consider the subdifferentials of $G_{1}, G_{2}$ with respect to the first variables.

Now we can show the main theorem of this section.

Theorem 3.3. The system (3.1)-(3.2) admits a solution $(s, p)$ in $\left[H_{0}^{1}(\Omega) \cap H^{2}(\Omega)\right]^{2}$.

Proof. By applying Theorem 3.2 we have a solution $(s, p)$ of (3.5) in $H_{0}^{1}(\Omega) \times H_{0}^{1}(\Omega)$. Now we calculate the subdifferentials of $G_{1}$ and $G_{2}$ actually and show that the solution of (3.5) satisfies System (3.1)(3.2).

We consider the functions $h_{1}, h_{2}, l_{1}$, and $l_{2}: \Omega \times R \rightarrow R$ defined by

$$
\begin{aligned}
& h_{1}(x, t)=\chi_{1}(x) \phi_{1}(t, p(x)), \\
& h_{2}(x, t)=\chi_{2}(x) \phi_{2}(t, p(x)), \\
& l_{1}(x, t)=\chi_{1}(x) \phi_{1}(t, s(x)),
\end{aligned}
$$

and

$$
l_{2}(x, t)=\chi_{2}(x) \phi_{2}(t, s(x)) .
$$

We need the following Lemma for the proof of Theorem. 
Lemma. $h_{i}, l_{i}, i=1,2$, satisfy the following conditions.

(a) $h_{i}(x, \cdot), l_{i}(x, \cdot)$ are proper, convex and continuous for each $x \in \Omega$.

(b) $h_{i}(\cdot, t) l_{i}(\cdot, t)$ are measurable for each $t \in R$.

(c) int $D_{h_{i}}(x) \equiv$ int $\left\{t \in R: h_{i}(x, t)<+\infty\right\}$ and int $D_{l_{i}}(x) \equiv$ int $\{t \in R$ : $\left.l_{i}(x, t)<+\infty\right\}$ are nonempty for each $x$ in $\Omega$.

(d) $h_{i}\left(x, u_{i}(x)\right)\left[r e s p . l_{i}\left(x, v_{i}(x)\right)\right]$ is majorized by a summable function of $x$ for at least one choice of $u_{i}\left[\right.$ resp. $\left.v_{i}\right]$ in $L^{2}(\Omega), i=1,2$.

(e) The conjugate function $h_{i}^{*}\left(x, u_{i}^{*}(x)\right)\left[\operatorname{resp} . l_{i}^{*}\left(x, v_{i}^{*}(x)\right)\right]$ of $h_{i}$ [resp. $\left.l_{i}\right]$ is majorized by a summable function of $x$ for at least one $u_{i}^{*}\left[\right.$ resp. $\left.v_{i}^{*}\right]$ in $L^{2}(\Omega), i=1,2$, where the conjugate functions are given by

$$
\begin{array}{ll}
h_{i}^{*}\left(x, s^{*}\right)=\sup \left\{s^{*} t-h_{i}(x, t): t \in R\right\}, & s^{*} \in R, \\
l_{i}^{*}\left(x, r^{*}\right)=\sup \left\{r^{*} t-l_{i}(x, t): t \in R\right\}, & r^{*} \in R .
\end{array}
$$

Proof. (a), (b) and (c) are obvious. We shall show (d) and (e). Taking $u_{i}(x)=v_{i}(x)=0$, we obtain

$$
\begin{aligned}
& h_{1}(x, 0)=-\sigma_{1} \chi_{1}(x)\left(1+\alpha p(x)^{+}\right) \log \left(1+\alpha p(x)^{+}\right), \\
& h_{2}(x, 0)=0, \\
& l_{1}(x, 0)=-\sigma_{1} \chi_{1}(x) \frac{s(x)^{+}}{\alpha} \log \left(1+s(x)^{+}\right),
\end{aligned}
$$

and

$$
l_{2}(x, 0)=0 \text {. }
$$

Thus (d) is satisfied. Take

$$
u_{1}^{*}(x)=0, u_{2}^{*}(x)=-\sigma_{2} \chi_{2}(x) \frac{p(x)^{+}}{1+\beta p(x)^{+}}, v_{1}^{*}(x)=-\sigma_{1} \chi_{1}(x) \frac{s(x)^{+}}{1+s(x)^{+}}
$$

and

$$
v_{2}^{*}(x)=0,
$$

then (e) is obtained by the following calculations.

$$
\begin{aligned}
& h_{1}^{*}(x, 0)=\sup _{t \in R}\left\{-h_{1}(x, t)\right\} \\
& =\sup \sigma_{1} \chi_{1}(x)\left\{\left(1+\alpha p(x)^{+}\right) \log \left(1+t^{+}+\alpha p(x)^{+}\right)-t^{+}\right\} \\
& \leqq \sigma_{1} \chi_{1}(x)\left(1+\alpha p(x)^{+}\right) \log \left(1+\alpha p(x)^{+}\right), \\
& h_{2}^{*}\left(x,-\sigma_{2} \chi_{2}(x) \frac{p(x)^{+}}{1+\beta p(x)^{+}}\right)
\end{aligned}
$$




$$
\begin{aligned}
&= \sup _{t \in R}\left\{-t \sigma_{2} \chi_{2}(x) \frac{p(x)^{+}}{1+\beta p(x)^{+}}+\sigma_{2} \chi_{2}(x) \frac{p(x)^{+}}{1+\beta p(x)^{+}} t\right\} \\
&= 0, \\
& l_{1}^{*}\left(x,-\sigma_{1} \chi_{1}(x) \frac{s(x)^{+}}{1+s(x)^{+}}\right) \\
&=\sup _{t \in R}\left[t\left\{-\sigma_{1} \chi_{1}(x) \frac{s(x)^{+}}{1+s(x)^{+}}\right\}+\sigma_{1} \chi_{1}(x)\left\{\frac{s(x)^{+}}{1+s(x)^{+}}\left(t-t^{+}\right)\right.\right. \\
&\left.\left.+\frac{s(x)^{+}}{\alpha} \log \left(1+\alpha t^{+}+s(x)^{+}\right)\right\}\right] \\
&= \sup _{t \in R} \sigma_{1} \chi_{1}(x)\left\{\frac{s(x)^{+}}{\alpha} \log \left(1+\alpha t^{+}+s(x)^{+}\right)-t^{+} \frac{s(x)^{+}}{1+s(x)^{+}}\right\} \\
& \leqq \sigma_{1} \chi_{1}(x) \frac{s(x)^{+}}{\alpha} \log \left(1+s(x)^{+}\right), \\
& l_{2}^{*}(x, 0)= \sup _{t \in R}\left\{-l_{2}(x, t)\right\} \\
&= \sup _{t \in R} \frac{\sigma_{2}}{\beta} \chi_{2}(x)\left\{\frac{1}{\beta} \log \left(1+\beta t^{+}\right)-t^{+}\right\}
\end{aligned}
$$

$\leqq 0$.

Now we return to the proof of Theorem. By using the results of Rockafellar [13], [14] and the above Lemma we obtain

$$
w_{i}^{*} \in \partial I_{h_{i}}(s) \quad\left[\text { resp. } z_{i}^{*} \in \partial I_{l_{i}}(p)\right]
$$

if and only if

$$
w_{i}^{*}(x) \in \partial h_{i, x}(s(x))\left[\operatorname{resp} . z_{i}^{*}(x) \in \partial l_{i, x}(p(x))\right]
$$

for a. e. $x \in \Omega, i=1,2$, where

$$
\begin{aligned}
& I_{h_{i}}(s)=\int_{\Omega} h_{i}(x, s(x)) d x \\
& I_{l_{i}}(p)=\int_{\Omega} l_{i}(x, p(x)) d x \\
& h_{i, x}(\cdot)=h_{i}(x, \cdot)
\end{aligned}
$$

and

$$
l_{i, x}(\cdot)=l_{i}(x, \cdot)
$$

By calculating the ordinary differentials, we have

$$
\frac{\partial h_{1}}{\partial t}(x, t)=\sigma_{1} \chi_{1}(x) \frac{t^{+}}{1+\alpha p(x)^{+}+t^{+}},
$$




$$
\begin{aligned}
& \frac{\partial h_{2}}{\partial t}(x, t)=-\sigma_{2} \chi_{2}(x) \frac{p(x)^{+}}{1+\beta p(x)^{+}} \\
& \frac{\partial l_{1}}{\partial t}(x, t)=-\sigma_{1} \chi_{1}(x) \frac{s(x)^{+}}{1+\alpha t^{+}+s(x)^{+}}
\end{aligned}
$$

and

$$
\frac{\partial l_{2}}{\partial t}(x, t)=\sigma_{2} \chi_{2}(x) \frac{t^{+}}{1+\beta t^{+}} \quad \text { for a. e. } x \in \Omega
$$

So we have

$$
\begin{aligned}
& \partial h_{1, x}(s(x))=\sigma_{1} \chi_{1}(x) \frac{s(x)^{+}}{1+\alpha p(x)^{+}+s(x)^{+}}, \\
& \partial h_{2, x}(s(x))=-\sigma_{2} \chi_{2}(x) \frac{p(x)^{+}}{1+\beta p(x)^{+}}, \\
& \partial l_{1, x}(p(x))=-\sigma_{1} \chi_{1}(x) \frac{s(x)^{+}}{1+\alpha p(x)^{+}+s(x)^{+}}
\end{aligned}
$$

and

$$
\partial l_{2, x}(p(x))=\sigma_{2} \chi_{2}(x) \frac{p(x)^{+}}{1+\beta p(x)^{+}} .
$$

By calculating the subdifferentials of the other terms, $\int|\nabla s|^{2}, \int f_{1} s, \int|\nabla p|^{2}$, $\int f_{2} p$ (see proposition 2.7, 2.8 of chapter 2 in Barbu [2]), we can show that the subdifferentials of $G_{1}, G_{2}$ are single valued and correspond to the terms in (3.1). Thus we have a solution $(s, p)$ in $\left[H_{0}^{1}(\Omega)\right]^{2}$ which satisfy (3.1)-(3.2). By using the arguments about the smoothness of solutions of regular elliptic problems in [9] (p. 212) we have

$$
s, p \in H_{0}^{1}(\Omega) \cap H^{2}(\Omega) .
$$

Q. E. D.

Remark. When the system depends to time-variables, it is important to analyze the time-dependent behavior of solutions in relation to the solutions in equilibrium. Under suitable boundary conditions, such as convergency and periodicity, we show the convergence to the equilibrium states ([5]) and the periodicity ([12]) of solutions of reaction-diffusion systems. 


\section{§4. Neumann Boundary Conditions}

We consider the constant flow of the substrate $S$ and the product $P$ through the boundary $\Gamma$ and assume that $S$ and $P$ are dissolved at the rate proportional to their concentration, $k_{1} s(x), k_{2} p(x)$ at $x$ in $\Omega$, where $k_{1}, k_{2}$ are positive constants. Then the system is given by

$$
\left\{\begin{array}{l}
-\Delta s+k_{1} s+\chi_{1} \frac{\sigma_{1} s^{+}}{1+\alpha p^{+}+s^{+}}-\chi_{2} \frac{\sigma_{2}}{1+\beta p^{+}}-f_{1}=0 \\
-\lambda \Delta p+k_{2} p+\chi_{2} \frac{\sigma_{2} p^{+}}{1+\beta p^{+}}-\chi_{1} \frac{\sigma_{1} s^{+}}{1+\alpha p^{+}+s^{+}}-f_{2}=0
\end{array}\right.
$$
in $\Omega$,

(4. 2) $\left\{\begin{array}{l}\frac{\partial s}{\partial n}=g_{1} \\ \frac{\partial p}{\partial n}=g_{2}\end{array}\right.$

where $g_{1}, g_{2} \in H^{ \pm}(\Gamma), \frac{\partial}{\partial n}$ is the normal derivative taken toward the exterior of $\Omega$, and we use the same notations as those in $\S 3$.

In order to use the Q.V.I. method, we consider the functions $J_{1}, J_{2}: H^{1}(\Omega) \times L^{2}(\Omega) \rightarrow R$ defined by

$$
\begin{aligned}
J_{1}(s, p)= & \frac{1}{2} \int_{\Omega}|\nabla s|^{2} d x+\frac{k_{1}}{2} \int_{\Omega}|s|^{2} d x+\int_{\Omega} \chi_{1} \phi_{1}(s, p) d x \\
& +\int_{\Omega} \chi_{2} \phi_{2}(s, p) d x-\int_{\Omega} f_{1} s d x-\int_{\Gamma} g_{1} s d y, \\
J_{2}(q, r)= & \frac{\lambda}{2} \int_{\Omega}|\nabla q|^{2} d x+\frac{k_{2}}{2} \int_{\Omega}|q|^{2} d x+\int_{\Omega} \chi_{1} \phi_{1}(q, r) d x \\
& +\int_{\Omega} \chi_{2} \psi_{2}(q, r) d x-\int_{\Omega} f_{2} q d x-\int_{\Gamma} g_{2} q d y
\end{aligned}
$$

where $\phi_{1}, \phi_{2}, \phi_{1}$, and $\phi_{2}$ are defined in $\S 3$.

Theorem 4.1. The system of the following Q.V.I. admits a solution $(s, p)$ in $H^{1}(\Omega) \times H^{1}(\Omega)$.

$$
\begin{cases}J_{1}(s, p) \leqq J_{1}(u, p) & \text { for all } u \text { in } H^{1}(\Omega) \\ J_{2}(p, s) \leqq J_{2}(v, s) & \text { for all } v \text { in } H^{1}(\Omega) .\end{cases}
$$


Proof. Let $U=M_{1}=M_{2}=H^{1}(\Omega), V=L^{2}(\Omega), F_{1}=J_{1}$, and $F_{2}=J_{2}$ in $\S 2$. By using the argument similar to $\S 3$ and applying Theorem 2. 1, it may be sufficient to show that $J_{1}, J_{2}$ satisfy the coercive condition (iii), but it is immediately clear from the calculations in $\S 3$ and the inequalities

$$
\begin{aligned}
& \frac{1}{2} \int|\nabla s|^{2}+\frac{k_{1}}{2} \int|s|^{2} \geqq \frac{1}{2} \min \left(1, k_{1}\right)|| s \|_{H^{1}(\Omega)}^{2}, \\
& \frac{\lambda}{2} \int|\nabla q|^{2}+\frac{k_{2}}{2} \int|q|^{2} \geqq\left.\frac{1}{2} \min \left(\lambda, k_{2}\right)|| q\right|_{H^{1}(\Omega)} ^{2} .
\end{aligned}
$$

Q. E. D.

From the definition of subdifferentials we have the following Theorem.

Theorem 4.2. We have a solution $(s, p)$ in $H^{1}(\Omega) \times H^{1}(\Omega)$ which satisfies

$$
\left\{\begin{array}{l}
0 \in \partial J_{1}(s, p) \\
0 \in \partial J_{2}(p, s)
\end{array}\right.
$$

where the subdifferentials with respect to the first variables are considered.

Definitions. $\langle\cdot, \cdot\rangle$ denotes the dual pairing between $H^{1}(\Omega)$ and $\left[H^{1}(\Omega)\right]^{\prime}$ and $\langle\cdot, \cdot\rangle_{\gamma}$ denotes the dual pairing between $H^{-\frac{1}{2}}(\Gamma)$ and $H^{\frac{1}{2}}(\boldsymbol{\Gamma})$. The inner products on $L^{2}(\Omega), L^{2}(\Gamma)$ are denoted by $(\cdot, \cdot)$, $(\cdot,)_{r}$, respectively.

$I, I_{0}$ are the continuous injections from $H^{1}(\Omega)$ into $L^{2}(\Omega)$, from $H^{\frac{1}{2}}(\Gamma)$ into $L^{2}(\Gamma)$, respectively, and $\gamma$ is the trace operator. Their dual operators are denoted by $I^{*}, I_{0}^{*}$ and $\gamma^{*}$.

Theorem 4.3. The system (4.1)-(4.2) admits a solution $(s, p)$ in $H^{2}(\Omega) \times H^{2}(\Omega)$.

Proof. By calculating the subdifferentials of $J_{1}, J_{2}$ and applying Theorem 4.2, we have

$$
\begin{array}{r}
\left\langle-\Delta s+I^{*}\left\{k_{1} s+\chi_{1} \frac{\sigma_{1} s^{+}}{1+\alpha p^{+}+s^{+}}-\chi_{2} \frac{\sigma_{2} p^{+}}{1+\beta p^{+}}-f_{1}\right\}\right. \\
\left.-\gamma^{*} I_{0}^{*} g_{1}, v\right\rangle=0 \text { for all } v \text { in } H^{1}(\Omega) .
\end{array}
$$

By Green's formula we obtain 


$$
\begin{gathered}
\left(-\Delta s+k_{1} s+\chi_{1} \frac{\sigma_{1} s^{+}}{1+\alpha p^{+}+s^{+}}-\chi_{2} \frac{\sigma_{2} p^{+}}{1+\beta p^{+}}-f_{1}, v\right) \\
+\left\langle\frac{\partial s}{\partial n}-g_{1}, v\right\rangle_{r}=0 \quad \text { for all } v \in H^{1}(\Omega) .
\end{gathered}
$$

Taking $v=\omega \in \mathscr{D}(\Omega)$ in the above equation, we have

$$
\begin{aligned}
\left(-\Delta s+k_{1} s+\chi_{1} \frac{\sigma_{1} s^{+}}{1+\alpha p^{+}+s^{+}}-\chi_{2} \frac{\sigma_{2} p^{+}}{1+\beta p^{+}}-f_{1}, \omega\right) & =0 \\
& \text { for all } \omega \in \mathscr{D}(\Omega) .
\end{aligned}
$$

Since $\mathscr{D}(\Omega)$ is dense in $L^{2}(\Omega)$, we have

$$
-\Delta s+k_{1} s+\chi_{1} \frac{\sigma_{1} s^{+}}{1+\alpha p^{+} s^{+}}-\chi_{2} \frac{\sigma_{2} p^{+}}{1+\beta p^{+}}-f_{1}=0 \quad \text { a. e. in } \Omega \text { 。 }
$$

Thus we have

$$
\left\langle\frac{\partial \mathrm{s}}{\partial n}-g_{1}, \gamma v\right\rangle_{r}=0 \quad \text { for all } v \text { in } H^{1}(\Omega) .
$$

It implies that

$$
\frac{\partial s}{\partial n}=g_{1} \quad \text { a. e. on } \Gamma \text {. }
$$

By the same argument about $J_{2}$, we obtain

$$
-\dot{\lambda} \Delta p+k_{2} p+\chi_{2} \frac{\sigma_{2} p^{+}}{1+\beta p^{+}}-\chi_{1} \frac{\sigma_{1} s^{+}}{1+\alpha p^{+}+s^{+}}-f_{2}=0 \quad \text { a. e. in } \Omega
$$

and

$$
\frac{\partial p}{\partial n}=g_{2} \quad \text { a. e. on } \Gamma \text {. }
$$

By using the argument about the regularity of regular elliptic problems in [9] (p. 212), we have $s, p$ in $H^{2}(\Omega)$.

Q.E.D.

\section{§5. A Priori Feedback Control}

Now we consider "a priori feedback control" model. We start from the theorem of Mossino about Q.V.I. Let $U, V$ be real reflexive Banach spaces and assume that $U$ is compactly imbedded in $V$. Let $K$ be a function defined on $U \times V$ with values in $(-\infty,+\infty]$ and assume that $K$ satisfies the following conditions (I), (II) and (III). 
(I) $K(\cdot, \cdot)$ is lower semi-continuous on $U$ (weak) $\times V$ (strong). For each $s$ in $U, K(s, \cdot)$ is continuous on $V$ (strong).

(II) For each $p$ in $V, K(\cdot, p)$ is proper, strictly convex.

(III) $K$ is coercive in the following sense; there exists an element $s_{0}$ in $U$ and a constant $m_{0}>0$ such that $K\left(s_{0}, p\right)<+\infty$ for each $p$ in $V$ and, if $\|s\|_{U}>m_{0}$,

$$
K(s, p)-K\left(s_{0}, p\right) \geqq 0 \quad \text { for all } p \text { in } V .
$$

The above coercive condition (III) is weaker than (iii) in $\S 2$. Furthermore, this condition can be weakened to the type which appeared in [15]. The following theorem is proved by using the same method as the proof Theorem 2.1.

Theorem 5.1. Under the above assumptions there exists $u$ in $U$ such that

$$
K(u, u) \leqq K(v, u) \quad \text { for all } v \text { in } V \text {, }
$$

that is,

$$
0 \in \partial K(u, u)
$$

where the subdifferential is taken with respect to the first variable.

First we consider the following system.

$$
-\Delta u+k u=f \quad \text { in } \Omega
$$

with the boundary condition

$$
\begin{cases}\frac{\partial u}{\partial n}=0 & \text { on } \Gamma_{+}=\left\{y \in \Gamma:\left.u\right|_{\Gamma}(y)>Q\left(\left.u\right|_{\Gamma}\right)(y)\right\} \\ \frac{\partial u}{\partial n}=m & \text { on } \Gamma_{-}=\left\{y \in \Gamma:\left.u\right|_{\Gamma}(y)<Q\left(\left.u\right|_{\Gamma}\right)(y)\right\} \\ 0 \leqq \frac{\partial u}{\partial n} \leqq m & \text { on } \Gamma_{0}=\Gamma-\Gamma_{+} \cup \Gamma_{-}\end{cases}
$$

where $k, m$ are positive constants and $f \in L^{2}(\Omega)$ and $Q: L^{2}(\Gamma) \rightarrow L^{2}(\Gamma)$ is continuous and $\sup _{w \in L^{2}(\Gamma)}|Q(w)|<+\infty$.

Take $U=H^{1}(\Omega)$ and $V=H^{\varepsilon}(\Omega)$ where $\varepsilon$ is a positive constant such that $\frac{1}{2}<\varepsilon<1$, then $U$ is compactly imbedded in $V$. We shall deal 
with this system by considering the function $K: H^{1}(\Omega) \times H^{\varepsilon}(\Omega) \rightarrow R$ defined by

$$
\begin{aligned}
K(u, v) & =\frac{1}{2} \int_{\Omega}|\nabla u|^{2} d x+\frac{k}{2} \int_{\Omega}|u|^{2} d x \\
& +m \int_{\Gamma}\left\{\left.u\right|_{\Gamma}-Q\left(\left.v\right|_{\Gamma}\right)\right\}-d y-\int_{\Omega} f u d x .
\end{aligned}
$$

When $v \in H^{\varepsilon}(\Omega), \frac{1}{2}<\varepsilon<1$, the trace of $v,\left.v\right|_{\Gamma}$ is in $H^{\varepsilon-\frac{1}{2}}(\Gamma)$. Since $Q: L^{2}(\Gamma) \rightarrow L^{2}(\Gamma)$ is continuous, if $v_{n} \rightarrow v$ in $H^{\varepsilon}(\Omega)$ as $n \rightarrow+\infty$, we have $Q\left(\left.v_{n}\right|_{\Gamma}\right) \rightarrow Q\left(\left.v\right|_{\Gamma}\right)$ in $L^{2}(\Gamma)$. Thus, by using the same argument as the proof of Theorem 3.1 (i), it follows that $K$ satisfies the condition (I). As for the condition (II), since the operator on $R ; t \rightarrow\{t\}^{-}$is convex, we can use the argument of the proof of Theorem 3.1 (ii). For the condition (III), take $s_{0}=0$, then by using the uniform boundedness of $Q$ we have

$$
\begin{aligned}
K(s, p) & -K(0, p)=\frac{1}{2} \int_{\Omega}|\nabla s|^{2} d x+\frac{k}{2} \int_{\Omega}|s|^{2} d x+m \int_{\Gamma}\left[\left\{\left.s\right|_{\Gamma}\right.\right. \\
& \left.\left.-Q\left(\left.p\right|_{\Gamma} \mid\right)\right\}^{-}-\left\{-Q\left(\left.p\right|_{\Gamma}\right)\right\}^{-}\right] d y-\int_{\Omega} f s d x \\
& \geqq\left. c_{1}|| s\right|_{H^{1}(\Omega)} ^{2}-m \int_{\Gamma}\left\{-Q\left(\left.p\right|_{\Gamma}\right)\right\}^{-} d y-\left.c_{2}|| s\right|_{H^{1}(\Omega)} \\
& \geqq\left. c_{1}|| s\right|_{H^{1}(\Omega)} ^{2}-c_{2}|| s \|_{H^{1}(\Omega)}-c_{3}
\end{aligned}
$$

where $c_{1}, c_{2}$ and $c_{3}$ are positive constants.

Thus there exist a constant $m_{0}>0$ which satisfies the condition (III).

Theorem 5.2. The system (5.3)-(5.4) admits a solution $u$ in $H^{1}(\Omega)$.

Proof. It is sufficient to calculate the subdifferential of $K$. By using the argument in the proof of Theorem 3.3 and 4.3 we obtain

$$
0 \in\left\langle-\Delta u+k u-f+m \gamma^{*} I_{0}^{*} \partial \int_{\Gamma}\{r u-Q(\gamma u)\}^{-}, w\right\rangle
$$

for all $w$ in $H^{1}(\Omega)$. Taking $w=\omega \in \mathscr{D}(\Omega)$ in the above equation, we obtain

$$
-\Delta u+\mathrm{k} u=f
$$

in the sense of distribution. By Green's formula, we have 


$$
0 \in(-\Delta u+k u-f, w)+\left\langle\frac{\partial u}{\partial n}+m I_{0}^{*} \partial \int_{\Gamma}\{\gamma u-Q(\gamma u)\}^{-}, \gamma w\right\rangle_{r}
$$

for all $w$ in $H^{1}(\Omega)$. And hence

$$
0 \in\left\langle\frac{\partial u}{\partial n}+m I_{0}^{*} \partial \int_{\Gamma}\{\gamma u-Q(\gamma u)\}^{-}, \gamma w\right\rangle_{r} .
$$

Let $\phi$ be a convex function from $L^{2}(\Gamma)$ to $R$ defined by

$$
\phi(v)=\int_{\Gamma}\{v\}^{-} d y, \quad v \text { in } L^{2}(\Gamma),
$$

then it is easily known that

$$
\partial \phi(u)= \begin{cases}0 & \text { on }\left\{y \in \Gamma:\left.u\right|_{\Gamma}>0\right\} \\ -1 & \text { on }\left\{y \in \Gamma:\left.u\right|_{\Gamma}<0\right\} \\ {[-1,0]} & \text { otherwise. }\end{cases}
$$

Thus we conclude that

$$
\begin{cases}\frac{\partial u}{\partial n}=0 & \text { on } \Gamma_{+} \\ \frac{\partial u}{\partial n}=m & \text { on } \Gamma_{-} \\ 0 \leqq \frac{\partial u}{\partial n} \leqq m & \text { on } \Gamma_{0} .\end{cases}
$$

Q. E. D.

Now we can study the reaction diffusion system with a priori feedback control by applying the previous results. We consider the system (4.1) with the following boundary conditions

(5.6) $\begin{cases}\frac{\partial s}{\partial n}=0 & \text { on }\left\{y \in \Gamma: \gamma s>Q_{1}(\gamma p)\right\} \\ \frac{\partial s}{\partial n}=m_{1} & \text { on }\left\{y \in \Gamma: \gamma s<Q_{2}(\gamma p)\right\} \\ 0 \leqq \frac{\partial s}{\partial n} \leqq m_{1}, & \end{cases}$
(5. 7) $\begin{cases}\frac{\partial p}{\partial n}=0 & \text { on }\left\{\nu \in \Gamma: \gamma p>Q_{2}(\gamma s)\right\} \\ \frac{\partial p}{\partial n}=m_{2} & \text { on }\left\{y \in \Gamma: \gamma p<Q_{2}(\gamma s)\right\} \\ 0 \leqq \frac{\partial p}{\partial n} \leqq m_{2} . & \end{cases}$ 
In (5.6) and (5.7) $m_{1}, m_{2}$ are positive constants, $Q_{i}: L^{2}(\Gamma) \rightarrow L^{2}(\Gamma)$ are continuous and $\sup _{w \in L^{2}(\Gamma)}\left|Q_{i}(w)\right|<+\infty, i=1,2$. By these functions $Q_{1}, Q_{2}$, the flow of each substance through the boundary is controlled according to the boundary value of the other chemical component.

Define $F_{1}, F_{2}: H^{1}(\Omega) \times H^{\varepsilon}(\Omega) \rightarrow R$ as follows;

$$
\begin{aligned}
F_{1}(s, p)= & \frac{1}{2} \int_{\Omega}|\nabla s|^{2} d x+\frac{k_{1}}{2} \int_{\Omega}|s|^{2} d x+\int_{\Omega} \chi_{1} \phi_{1}(s, p) d x \\
& +\int_{\Omega} \chi_{2} \phi_{2}(s, p) d x+m_{1} \int_{\Gamma}\left\{\gamma s-Q_{1}(\gamma p)\right\}-d y-\int_{\Omega} f_{1} s d x \\
F_{2}(q, r)= & \frac{1}{2} \int_{\Omega}|\nabla q|^{2} d x+\frac{k_{2}}{2} \int_{\Omega}|q|^{2} d x+\int_{\Omega} \chi_{1} \phi_{1}(q, r) d x \\
& +\int_{\Omega} \chi_{2} \psi_{2}(q, r) d x+m_{2} \int_{\Gamma}\left\{\gamma q-Q_{2}(\gamma r)\right\}-d y-\int_{\Omega} f q d x
\end{aligned}
$$

where $\phi_{1}, \phi_{2}, \phi_{1}, \phi_{2}$ are defined in $\S 3$. Then, by Theorem 2.1 and the previous arguments, we obtain the following theorem.

Theorem 5.3. System $(4.1)-(5.6)-(5.7)$ admits a solution $(s, p)$ in $H^{1}(\Omega) \times H^{1}(\Omega)$.

Remark. Enzymatically catalyzed membranes are of primary importance in various biological process, because they control the flow of chemical substances ([16]). Mechanism of this control is very complicated, but the recent progress in biochemistry shows that proteins in membrane play the most important role by catalyzing the reaction which occurrs active transport. In our models the functions $Q_{1}$ and $Q_{2}$ correspond to this control. To give an actual form of $Q_{i}$ in each biochemical model will be a complicated, but an interesting problem.

\section{Acknowledgment}

The author wishes to express his gratitude to Professor W. Takahashi for his valuable comments and to the referee for his careful reading and many useful suggestions. 


\section{References}

[1] Banks, H. T., Modeling of control and dynamical systems in the life sciences, in Optimal Control Theory and its Applications, Part I, Lecture Notes iu Economics and Mathematical Systems 105, Springer, (1974), 1-112.

[2] Barbu, V. and Precupanu, T. "Convexity and Optimization in Banach Space", Editura Academi, Sijithoff and Noordhoff, Romania, 1978.

[3] Duvaut, G. and Lions, J. L., "Sur les inéquations en mécanique et en physique”, Dunod, Paris, 1972.

[4] Ekeland, I. and Temam, R., “Analyse Convexe et Problem $\bullet$ Variationnels", Paris, DunodGauthier Villars, 1973.

[5] Hirano, N. and Naito, K., On the asymptotic behavior of solutions of a reaction diffusion system, to appear in J. Math. Anal. Appl.

[6] Kellog, R. B., Uniqueness in the Schauder fixed point theorem, Proc. Amer. Math. Soc. 60 (1976), 207-210.

[7] Kernevez, J. P., Modeling, simulation, identification and optimal control of large biochemical systems, in 5th Conference on Optimization Techniques, Part II, Lecture Notes in Computer Science 4, Springer, 1973, 357-365.

[8] Lions, J. L., Various topics in the theory of optimal control of distributed systems, in Optimal Control Theory and its Applications, Part I, Lecture Notes in Economics and Mathematical Systems 105, Springer, 1974, 166-309.

[9] Lions, J. L. and Magenes, E., “Non-Homogeneous Boundary Value Problems and Applicatıons", Springer-Verlag, 1967.

[10] Mosco, U., Implicit Variational Problems and Quasi Variational Inequalities, in Nonlinear Operators and the Calculus of Variations, Bruxelles 1975, Springer Lecture Notes in Math. 543, 1976, 83-156.

[11] Mossino, J., Etude de quelques non linéaires d'un type nouveau apparaissant en physique des plasmas, Publications Mathematiques d'Orsay, 1977.

[12] Naito, K., On the almost periodicity of solutions of a reaction diffusion system, $J$. Differential Equations 44 (1982), 9-20.

[13] Rockafellar, R. T., Integrals which are convex functionals I, Pacific J. Math. 24 (1968), 525-539.

[14] Integrals which are convex functionals II, Pacific J. Math. 39 (1971), 439-469.

[15] Takahashi, W., Nonlinear variational inequalities and fixed point theorems, J. Math. Soc. Japan 28 (1976), 168-181.

[16] Tien, H. T., Bilayer Lipid Membranes Theory and Practice, Marcel Dekker Inc. New York, 1974. 